\title{
Differential Decline in Illicit Drug Use by Sexual Identity Among United States High School Students, 2005-2017
}

\author{
Dylan Felt, ${ }^{1}$ Xinzi Wang, MA, ${ }^{1}$ Megan M. Ruprecht, ${ }^{1}$ Blair Turner, MPH, ${ }^{1}$ Lauren B. Beach, PhD/JD, \\ Morgan Mari Philbin, PhD, ${ }^{2}$ Michelle Birkett, PhD, and Gregory Phillips II, PhD, MS ${ }^{1}$
}

\begin{abstract}
Purpose: Adolescent drug use remains a significant public health concern. Sexual minority youth (SMY) are at elevated risk for illicit drug use compared with their heterosexual peers. We investigated this pattern at the national level, exploring whether trends and disparities in drug use vary over time and by sexual identity.

Methods: This study used Youth Risk Behavior Survey (YRBS) data, which were collected at seven time points from 2005 to 2017. Trends and disparities over time in the use of five drugs, as well as any drug use, were analyzed by self-reported sexual identity.

Results: The results demonstrated a general decrease in drug use behaviors from 2005 to 2017. The greatest number of significant decreases was among heterosexual and bisexual students; the fewest were among gay and lesbian students. Disparities between heterosexual youth and SMY persisted across years, and were greater for gay and lesbian students in 2017 than for bisexual and not-sure youth.

Conclusions: Our results represent the most comprehensive analysis of recent trends in drug use by sexual identity to date. Disparities in drug use remained significant despite overall downward trends within our sample. The significant decreases among bisexual students are not readily explained. We emphasize the need for ongoing research in this area, particularly given the currently volatile social position of sexual minority populations in the United States, and for culturally responsive and trauma-informed responses to SMY drug use.
\end{abstract}

Keywords: disparities, drug use, sexual minority, trends, youth, Youth Risk Behavior Survey

\section{Introduction}

D RUG USE AMONG adolescents remains a significant public health concern. 2019 data from the Monitoring the Future study estimated that $14.2 \%$ of high school-aged youth nationwide had used illicit drugs (excluding marijuana) in their lifetime, the same prevalence as in $2018 .{ }^{1}$ Adolescent drug use is associated with detrimental developmental and health risks that have implications across the lifecourse. ${ }^{2}$ Given these concerns, it is important to track trends in the prevalence of drug use over time to ensure that population-level patterns are well understood, especially for illicit drugs, including cocaine, inhalants, methamphetamines, heroin, and MDMA (methylenedioxymethamphetamine; more commonly known as "Ecstasy" or "Molly"). Through better understanding of populationlevel trends, we can ensure that targeted research approaches and intervention designs are responsive to areas of greatest overall need.
There are apparent disparities in drug use between sexual minority (e.g., lesbian, gay, bisexual [LGB]) and sexual majority (i.e., heterosexual) populations regardless of age. $^{3-11}$ Among youth, this disparity is particularly notable; one meta-analysis concluded that the odds of substance use were, overall, $190 \%$ higher for LGB youth; the effect size was even higher for some subpopulations, with sexual minority girls and bisexual individuals being particularly affected. ${ }^{5}$ These findings have been further confirmed in multiple cross-sectional and cohort studies, which found elevated levels of illicit drug use among sexual minority youth (SMY), including cocaine, ${ }^{3}$ ecstasy, ${ }^{3}$ misuse of prescription drugs, ${ }^{4}$ and polysubstance use. ${ }^{12}$

Minority Stress Theory (MST), originally developed to explain disparities in mental health among LGB populations, holds that experiences of stigma or discrimination on the basis of one's minority identity or identities produce mental and physical stress responses, which then lead to downstream health disparities among minority groups. ${ }^{13,14}$ The model

\footnotetext{
${ }^{1}$ Department of Medical Social Sciences, Northwestern University Feinberg School of Medicine, Chicago, Illinois, USA.

${ }^{2}$ Department of Sociomedical Sciences, Columbia University Mailman School of Public Health, New York, New York, USA.
} 
also illustrates that multilevel imbalances in social power and stigma exposure are important in determining health outcomes among sexual minority individuals. ${ }^{15}$ Consistent with this application, experiences of stigma and victimization have been consistently linked to negative health outcomes, including drug use and abuse among sexual minority individuals, particularly SMY. ${ }^{8,16,17}$

Despite these disparities, there is a paucity of comprehensive population research on drug use among SMY. Recent analyses of trends in SMY health behaviors have focused on alcohol, ${ }^{18}$ cigarette, ${ }^{19}$ and marijuana ${ }^{20}$ use resulting in a dearth of literature addressing other drug use behaviors. In addition, less work has explored these relationships over time. For example, Monitoring the Future, a national surveillance system of American adolescents, recently reported that youths' illicit drug use has remained largely stable since reaching the lowest levels seen in decades. ${ }^{21}$ In 1997, 30\% of 12th graders reported trying an illicit drug other than marijuana at some point in their lives. Lifetime prevalence fell to $24 \%$ by 2009 , and $19 \%$ by 2018 , although it remained stable in recent years. ${ }^{21}$ However, these analyses did not consider sexual orientation, masking key subpopulation risk. ${ }^{22}$

Describing longitudinal trends and drug use patterns among SMY on a national scale is thus helpful to accurately depict health disparities, evaluate the changing state of sexual minority health, inform research, and direct prevention and harm reduction efforts. Moreover, assessing the degree of differences between sexual minority and heterosexual students in the context of trend analyses is helpful to better understand whether trends represent meaningful changes in drug use disparities. ${ }^{19,23}$ Therefore, this study used pooled data from the biennial jurisdiction-level Youth Risk Behavior Survey (YRBS) to examine trends and disparities in drug use among sexual minority and heterosexual youth between 2005 and 2017.

\section{Methods}

\section{Data source}

The YRBS is a Centers for Disease Control and Prevention (CDC)-funded survey administered biennially to students in grades $9-12$ on national and local levels. ${ }^{24}$ To achieve a representative sample of students, jurisdictions use a two-stage cluster sample design. ${ }^{24} \mathrm{~A}$ new sample is selected each year; students are not followed over time. This study was reviewed by the Northwestern University Institutional Review Board and received a designation of "exempt" as it is based on secondary data analysis.

\section{Analytic sample}

Local YRBS data were pooled across multiple city and state level jurisdictions, biennially from 2005 to 2017, from sites that assessed sexual identity. The dataset consists of 52 total jurisdictions composed of 20 cities and 32 states across 7 time points, resulting in 156 jurisdiction years (529,999 students). For the present analysis, students were excluded if they were missing any primary demographic variables and all drug use variables (grade: $0.4 \%$; race/ethnicity: $3.2 \%$; sex: $0.8 \%$; drug use: $2.7 \%$; not mutually exclusive), resulting in a final sample of 508,539 participants.

\section{Measures}

Sex. Sex was determined by asking participants "What is your sex?" with the choices: (1) male or (2) female. The YRBS does not consistently assess gender identity, therefore we do not infer gender within our sample.

Sexual identity. Sexual identity was assessed by asking, "Which of the following best describes you?" Response options included: (1) heterosexual (straight); (2) gay or lesbian; (3) bisexual; and (4) not sure.

Race/ethnicity. Race/ethnicity was assessed using two questions. First, participants were asked if they identified as Hispanic or Latino (yes/no). Second, participants were asked to select all of the relevant races from the following list: American Indian or Alaska Native; Asian; Black or African American; Native Hawaiian or Other Pacific Islander; and White. Using the CDC's classification, these variables were combined into seven racial/ethnic groups: (1) American Indian or Alaska Native; (2) Asian; (3) Black or African American; (4) Hispanic/Latino (regardless of reported race); (5) Native Hawaiian/Other Pacific Islander; (6) White; and (7) Multiple Races (Non-Hispanic). Due to sample size, individuals who reported a Native Hawaiian/Other Pacific Islander, American Indian or Alaska Native, or Multiple Races identity were collapsed as "another race."

Grade. Participants were asked, "In what grade are you?" Reponse options were "9th," "10th," "11th," "12th," and "ungraded or other grade." Participants that selected "ungraded or other grade" were removed from analysis $(N=809 ; 0.1 \%)$.

Drug use. Drug use was assessed by asking "During your life, how many times have you used [drug]?" for 5 drugs. For cocaine (any form, including powder, crack, or freebase), inhalants (such as glue, aerosol, paints, etc.), methamphetamine, heroin, and MDMA, the response options ranged from, " 0 times" to " 40 or more times." Responses were dichotomized as yes/no for lifetime use of each drug. A composite variable was created to represent lifetime use of any drug-individuals who reported ever using any substance were coded "yes," individuals who did not were coded "no."

\section{Statistical analyses}

Data cleaning and recoding were conducted in SAS Version 9.4 (SAS Institute Inc., Cary, NC). Analyses were conducted using SAS-Callable SUDAAN Version 11.0.1 (RTI International, Research Triangle Park, NC) to appropriately weight estimates and to account for the YRBS' complex sampling design. The YRBS data weights adjust for student nonresponse and distribution of students by grade, sex, and race/ethnicity in each jurisdiction.

Descriptive analyses were conducted to determine weighted drug use prevalence by sex and sexual identity at each time point. ${ }^{25}$ Trends in drug use behaviors by sexual identity from 2005 to 2017 were assessed using the CDC's recommended approach to trend analysis; time was modeled as a continuous variable using orthogonal coefficients to reflect the biennial spacing of the surveys. ${ }^{26}$ Trend analyses 
were stratified by sex, controlled for grade, jurisdiction, and race/ethnicity, and assessed linear, quadratic, and cubic trends. The linear, cubic, and quadratic time component was considered significant at $p<0.05$. There were no significant cubic trends. The linear regression slope serves as a measure of the average increase or decrease over the 10 -year period, and whether the slope was significantly different from zero is a measure of whether the trend of drug use behaviors was, on average, upward or downward. Finally, to assess disparities by sexual identity among drug use by year, we used Fish and Baams' approach ${ }^{23}$ to conduct logistic regressions for each drug in each survey year to compare within-year drug use prevalence among sexual minority subgroups relative to heterosexual students.

\section{Results}

\section{Demographics}

The total weighted pooled sample across all years was $50.5 \%$ male and $49.5 \%$ female; the majority identified as White $(45.3 \%)$, followed by Hispanic (30.0\%), Black (14.9\%), Asian (4.9\%), and another race (4.9\%). Most reported a heterosexual identity $(87.0 \%)$ whereas $6.7 \%$ identified as bisexual, $3.9 \%$ as not sure, and $2.5 \%$ as gay/lesbian (Table 1).

\section{Drug use prevalence}

Just over $1 / 10$ th of students in all survey years reported any drug use (10.2\%) (Table 1). Inhalants were the most

Table 1. Sample Demographics and Substance Use Prevalence by Sex

\begin{tabular}{|c|c|c|c|c|c|c|}
\hline & \multicolumn{2}{|c|}{ Total } & \multicolumn{2}{|c|}{ Females } & \multicolumn{2}{|c|}{ Males } \\
\hline & $\mathrm{N}$ & Column \% & $\mathrm{n}$ & Column \% & $\mathrm{n}$ & Column \% \\
\hline Sexual identity & 506,367 & 100 & 260,424 & 100 & 245,943 & 100 \\
\hline Heterosexual & 444,045 & 86.98 & 217,714 & 82.66 & 224,928 & 91.44 \\
\hline Gay/Lesbian & 11,827 & 2.48 & 5755 & 2.25 & 5867 & 2.63 \\
\hline Bisexual & 32,062 & 6.65 & 24,986 & 10.31 & 6772 & 3.01 \\
\hline Not sure & 20,605 & 3.89 & 11,969 & 4.78 & 8376 & 2.93 \\
\hline Grade & 502,858 & 100 & 258,143 & 100 & 242,955 & 100 \\
\hline 9th & 137,401 & 27.03 & 70,531 & 26.71 & 66,418 & 27.35 \\
\hline 10th & 134,288 & 25.96 & 69,373 & 25.88 & 64,381 & 25.99 \\
\hline 11 th & 124,500 & 23.98 & 63,752 & 24.18 & 60,309 & 23.79 \\
\hline 12 th & 106,669 & 23.03 & 54,487 & 23.24 & 51,847 & 22.87 \\
\hline Race & 508,539 & 100 & 260,424 & 100 & 245,943 & 100 \\
\hline White & 217,845 & 45.28 & 110,580 & 45.37 & 106,455 & 45.28 \\
\hline Black & 76,456 & 14.86 & 40,112 & 15.09 & 36,068 & 14.63 \\
\hline Hispanic & 133,553 & 30.01 & 69,387 & 30.17 & 63,484 & 29.86 \\
\hline Asian & 36,810 & 4.92 & 18,299 & 4.63 & 18,381 & 5.20 \\
\hline Other & 43,875 & 4.92 & 22,046 & 4.74 & 21,555 & 5.04 \\
\hline Region & 494,381 & 100 & 253,052 & 100 & 239,217 & 100 \\
\hline Northeast & 264,758 & 26.28 & 135,151 & 26.38 & 128,318 & 26.21 \\
\hline Midwest & 59,025 & 18.45 & 30,549 & 18.51 & 28,283 & 18.43 \\
\hline South & 79,616 & 24.13 & 41,180 & 24.20 & 38,055 & 24.03 \\
\hline West & 90,982 & 31.15 & 46,172 & 30.91 & 44,561 & 31.33 \\
\hline Cocaine use & 392,393 & 100 & 202,918 & 100 & 188,014 & 100 \\
\hline Yes & 20,134 & 5.17 & 7660 & 3.85 & 12,154 & 6.34 \\
\hline No & 372,259 & 94.83 & 195,258 & 96.15 & 175,860 & 93.66 \\
\hline Inhalant use & 322,356 & 100 & 165,980 & 100 & 154,992 & 100 \\
\hline Yes & 26,854 & 7.31 & 13,589 & 7.34 & 12,929 & 7.14 \\
\hline No & 295,502 & 92.69 & 152,391 & 92.66 & 142,063 & 92.86 \\
\hline Meth use & 350,239 & 100 & 179,998 & 100 & 168,970 & 100 \\
\hline Yes & 11,275 & 2.87 & 3912 & 1.90 & 7131 & 3.68 \\
\hline No & 338,964 & 97.13 & 176,086 & 98.10 & 161,839 & 96.32 \\
\hline Heroin use & 381,856 & 100 & 196,765 & 100 & 183,663 & 100 \\
\hline Yes & 10,140 & 2.36 & 3048 & 1.31 & 6801 & 3.27 \\
\hline No & 371,716 & 97.64 & 193,717 & 98.69 & 176,862 & 96.73 \\
\hline MDMA $^{\mathrm{a}}$ use & 286,809 & 100 & 148228 & 100 & 137635 & 100 \\
\hline Yes & 16,159 & 5.32 & 6558 & 4.19 & 9376 & 6.24 \\
\hline No & 270,650 & 94.68 & 141670 & 95.81 & 12,8590 & 93.76 \\
\hline Any drug use & 490,620 & 100 & 252039 & 100 & 236,519 & 100 \\
\hline Yes & 50,576 & 10.16 & 23139 & 9.06 & 26,832 & 11.04 \\
\hline No & 440,044 & 89.84 & 228900 & 90.94 & 209,687 & 88.96 \\
\hline
\end{tabular}

aMethylenedioxymethamphetamine; more commonly known as "Ecstasy" or "Molly." MDMA was assessed by asking participants, "How many times have you used ecstasy (also called MDMA)?" 
frequently used substance (7.3\%), followed by MDMA $(5.3 \%)$, cocaine $(5.2 \%)$, meth $(2.9 \%)$, and heroin $(2.4 \%)$. Drug use also varied by sex, with drug use across all categories, except inhalants, more prevalent among male students than female students (Table 1). A total of $53.5 \%$ of bisexual students reported any drug use, much higher than gay/lesbian (28.0\%), not sure (20.6\%), or heterosexual (11.1\%) students. These differences varied by type of drug used. The prevalence of sexual identity and drug use by survey year is provided in Supplementary Tables S1 and S2.

\section{Drug use trends by sexual identity - males}

Bisexual and heterosexual males demonstrated the greatest number of significant linear decreases, observed in all drug use categories, except for heroin (Supplementary Table S3). Among not-sure male youth, cocaine (20092017) and MDMA (2013-2017) use decreased significantly with minor fluctuations year to year, but other drug use categories tended to fluctuate by year with overall nonsignificant decreases over time. No significant changes were observed among gay male youth. Trends are visualized in Figure 1.

\section{Drug use trends by sexual identity-females}

Among females, bisexual students demonstrated significant decreases across all substance use categories other than heroin use (Supplementary Table S4). Heterosexual students demonstrated an overall significant decrease in the use of all drugs over time with fluctuations; heroin use increased from 2005 to 2015 and decreased from 2015 to 2017. Other drugs demonstrated no change in early years, followed by a significant decrease (inhalants and any drug use decreased from 2009 to 2017 and MDMA decreased from 2013 to 2017). Lesbian and not-sure female youth demonstrated the fewest significant trends; among lesbian youth, only cocaine, MDMA, and any drug use showed significant decreases; however, all other drug use among lesbian youth demonstrated nonsignificant decreases. Among not-sure female youth, only cocaine, inhalants, and any drug use decreased significantly. Notably, there was a nearly $50 \%$ drop in any drug use between 2005 (21.0\%) and 2017 (9.8\%) among not-sure females (Supplementary Table S4). Trends are visualized in Figure 2.

\section{Within-year disparities by sexual identity-males}

Compared with heterosexual males, odds of drug use among sexual minority males were significantly elevated for all drugs in all years, except for cocaine use among not-sure males in 2005 and 2009, and for inhalant use among gay males in 2005 and not-sure males in 2009 (Table 2). In general, disparities between heterosexual and sexual minority males persisted yet fluctuated from year to year. For example, odds of cocaine use among gay males as compared with heterosexual males were higher in 2013 than in 2015 (adjusted odds ratio $[\mathrm{aOR}]=4.56,95 \%$ confidence interval [CI] 3.43-6.07 in 2013; aOR $=3.86,95 \% \mathrm{CI}$ $2.72-5.47$ in 2015). The same disparity was greater in $2017(\mathrm{aOR}=5.79,95 \%$ CI 4.62-7.26) than in 2013 or 2015 , almost the same in $2011(\mathrm{aOR}=3.90,95 \%$ CI 2.59 $5.85)$ as in 2015 , and narrower in $2009(\mathrm{aOR}=3.20,95 \%$
CI 1.81-5.65) than in 2011-2017. Caution should be exercised in interpreting these findings due to large CIs in certain drug use prevalence in certain years as a result of small sample sizes (Supplementary Table S3).

\section{Within-year disparities by sexual identity—females}

Similar to males, odds of drug use among sexual minority females were generally significantly elevated, although there were several instances of nonsignificant elevation in 2005 and 2007, and elevated odds of heroin use among bisexual females in 2011 were nonsignificant (Table 2). Disparities between sexual minority and heterosexual females also tended to fluctuate from year to year in a similar way as observed among males. In 2017, all disparities were significant, and were greater among lesbian females than among bisexual and not-sure females. Caution should be exercised in interpreting these findings due to large CIs in certain drug use prevalence in certain years as a result of small sample sizes (Supplementary Table S4).

\section{Discussion}

Our results present the most recent trend analysis of use of less common, illicit substances among U.S. youth by sex and sexual identity. We noted declines from 2005 to 2017 in all substance use regardless of sex and sexual identity, although not all declines were significant across groups. The greatest decreases were observed among heterosexual and bisexual students. Despite these decreases, disparities between heterosexual and SMY persisted, tended to fluctuate across all years, and were greatest among gay and lesbian youth in 2017, although odds were notably elevated among gay, lesbian, bisexual, and not-sure youth. Interpreted in light of MST, such results seem to indicate that overall levels of stigma and discrimination against SMY continue to have an impact on drug use despite broader downward trends in youth drug use nationally.

We also observed differential patterns of drug use both by year and over time within sexual minority subpopulations. In addition to demonstrating the fewest statistically significant decreases in drug use, gay and lesbian youth also demonstrated greater disparities in drug use than bisexual and not-sure youth as of 2017. Conversely, bisexual youth were the only sexual minority group to demonstrate a statistically significant decrease in all drug use categories regardless of sex, with the exception of heroin use. This observation was unexpected, and may be explained by bisexual students reporting greater prevalence of drug use initially than other sexual identity groups (Supplementary Tables S3 and S4), meaning that bisexual students may simply have had more opportunity for decrease than other groups. However, it was also notable that in the 2017 data, bisexual students demonstrated less elevated disparities as compared with gay and lesbian youth regardless of sex. This result is especially striking due to the significant body of prior research which highlights the uniquely elevated risk that bisexual people face for drug use and abuse. ${ }^{3,5,7}$

One prior study using the YRBS dataset has similarly found a "narrowing of disparities" among bisexual populations with regard to several domains of alcohol use. ${ }^{23}$ However, it should be noted that this study reported changes between two survey years only. Given the fluctuation in 
FIG. 1. Trends in drug use for heterosexual, gay, bisexual, and not-sure male youth, 2005-2017, pooled Youth Risk Behavior Survey dataset.

MDMA, methylenedioxymethamphetamine; more commonly known as "Ecstasy" or "Molly."

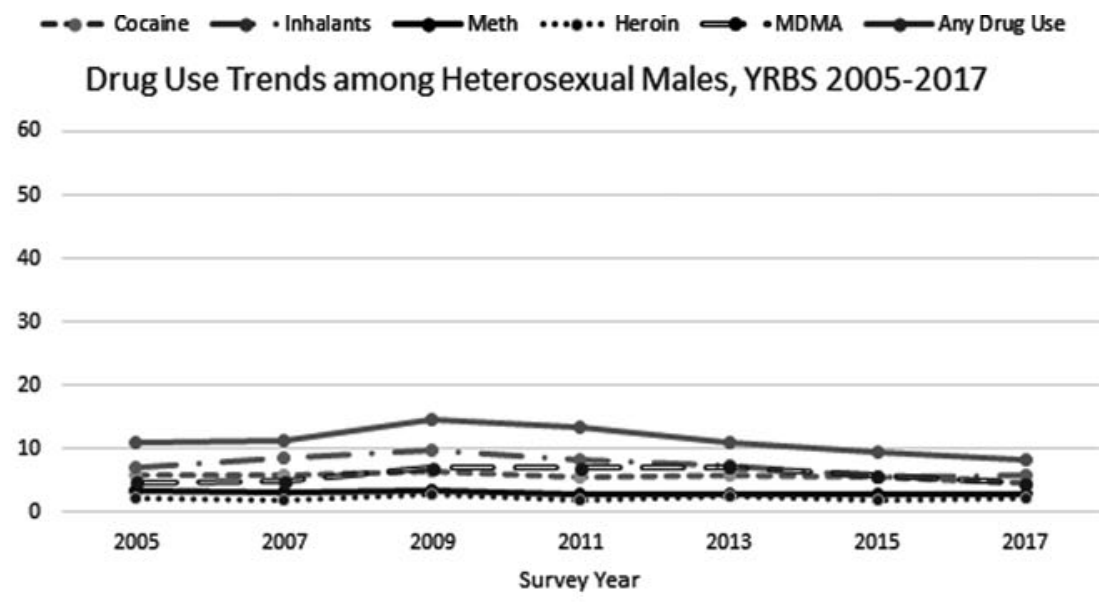

Drug Use Trends among Gay Males, YRBS 2005-2017
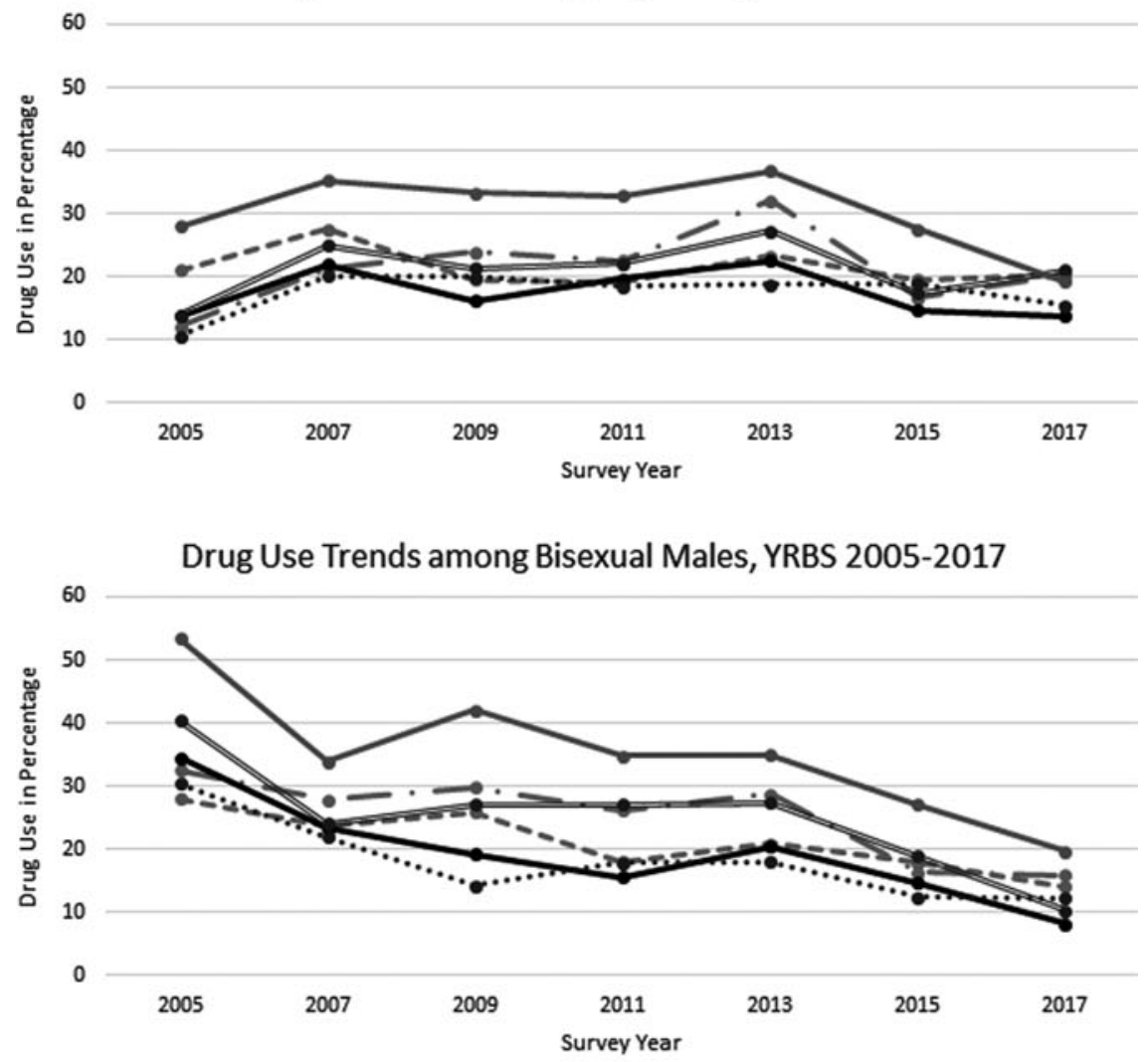

Drug Use Trends among Not Sure Males, YRBS 2005-2017

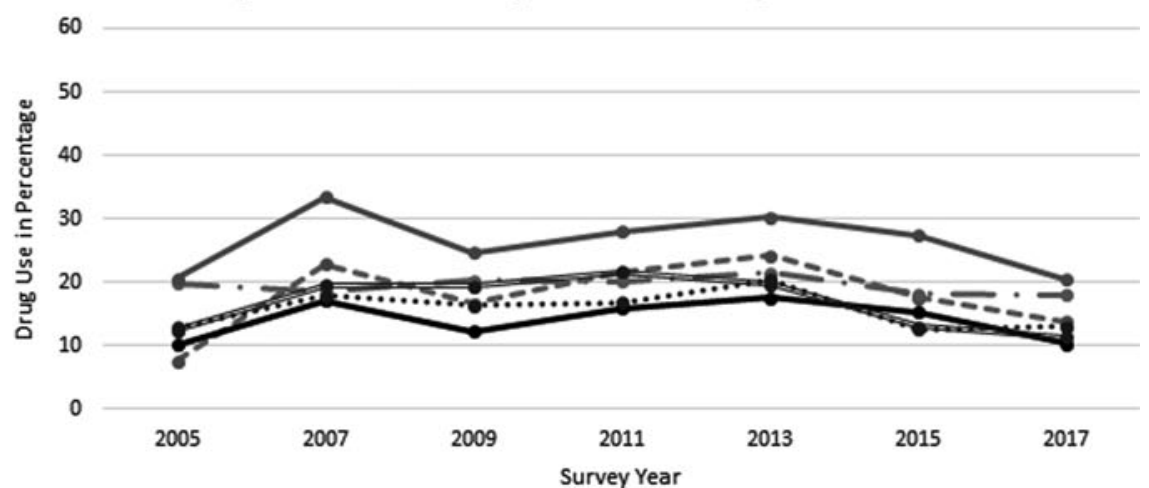




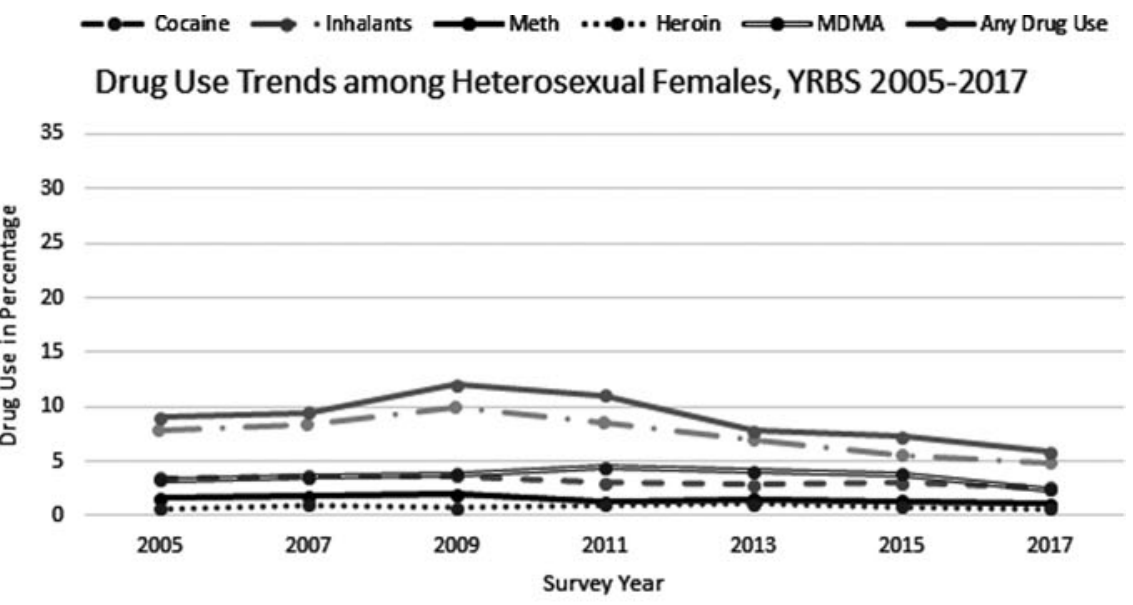

Drug Use Trends among Lesbian Females, YRBS 2005-2017

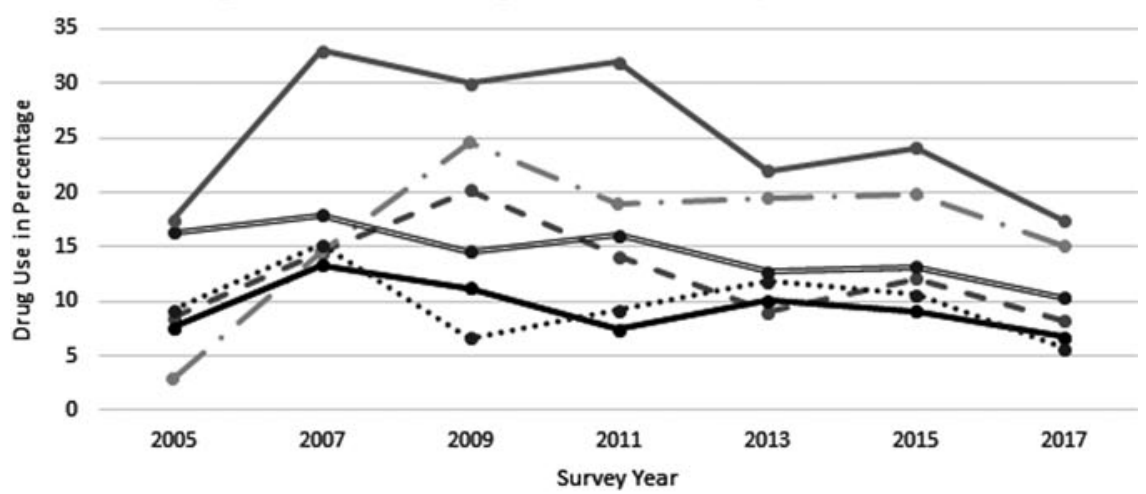

Drug Use Trends among Bisexual Females, YRBS 2005-2017

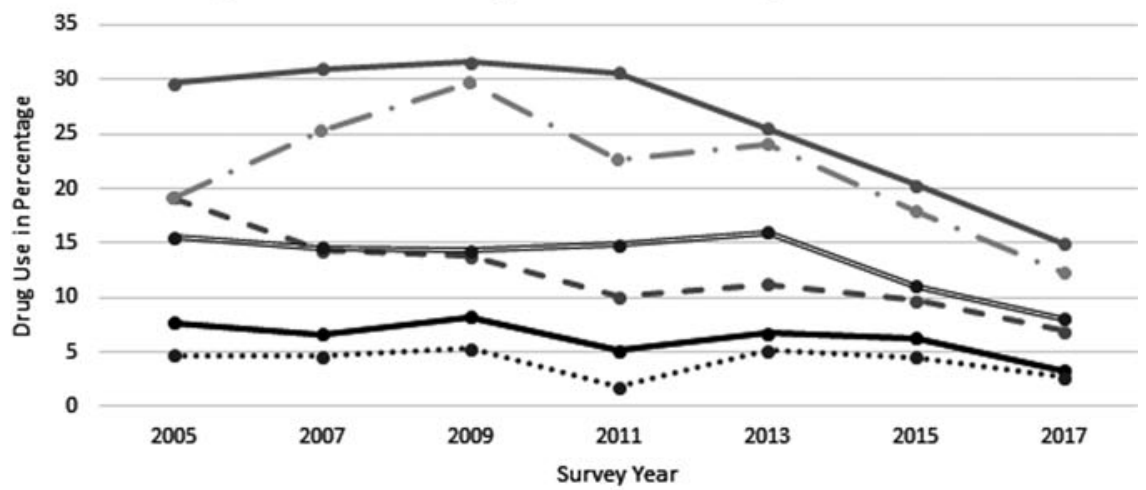

Drug Use Trends among Not Sure Females, YRBS 2005-2017

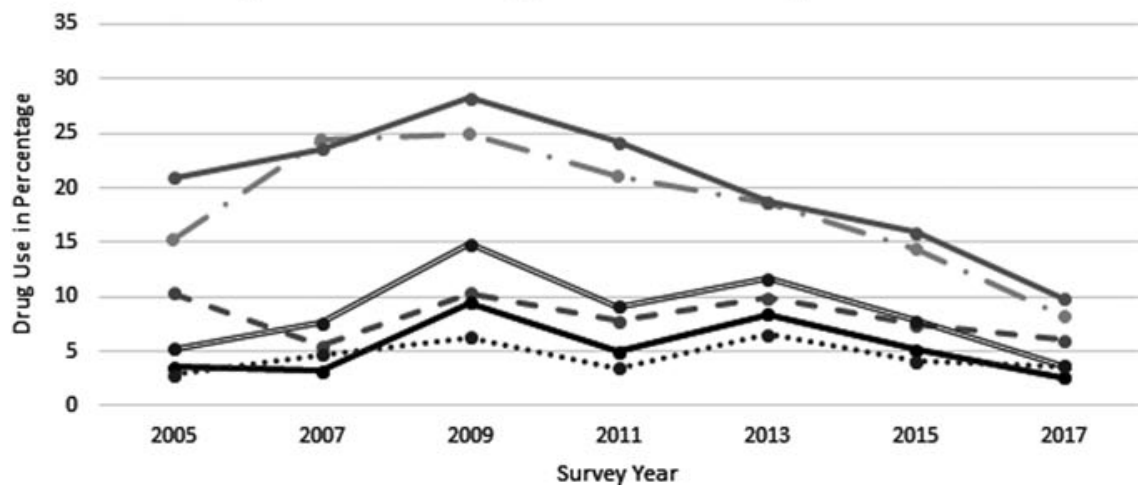

FIG. 2. Trends in drug use for heterosexual, lesbian, bisexual, and notsure female youth, 2005-2017, pooled Youth Risk Behavior Survey dataset. 


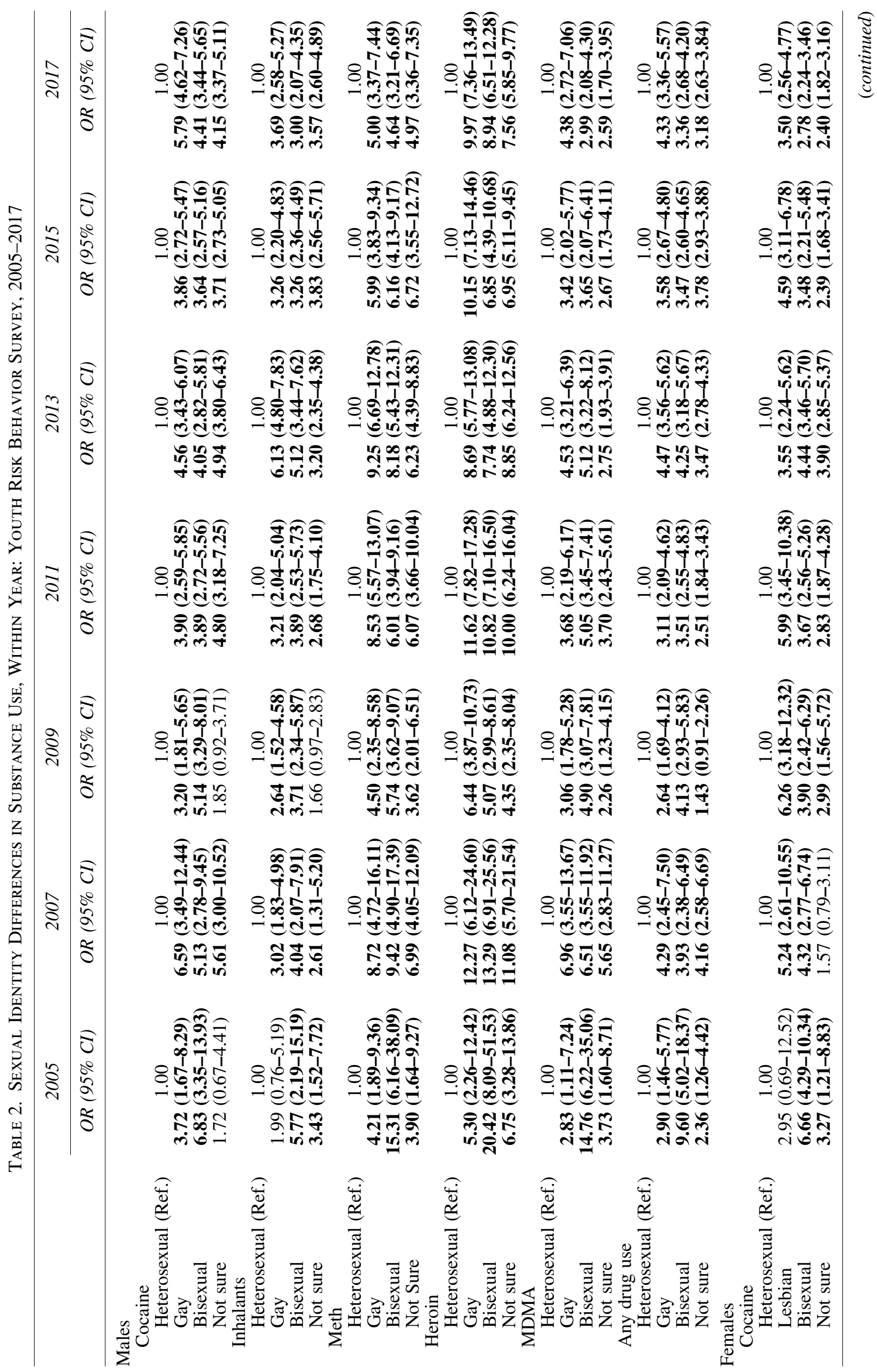




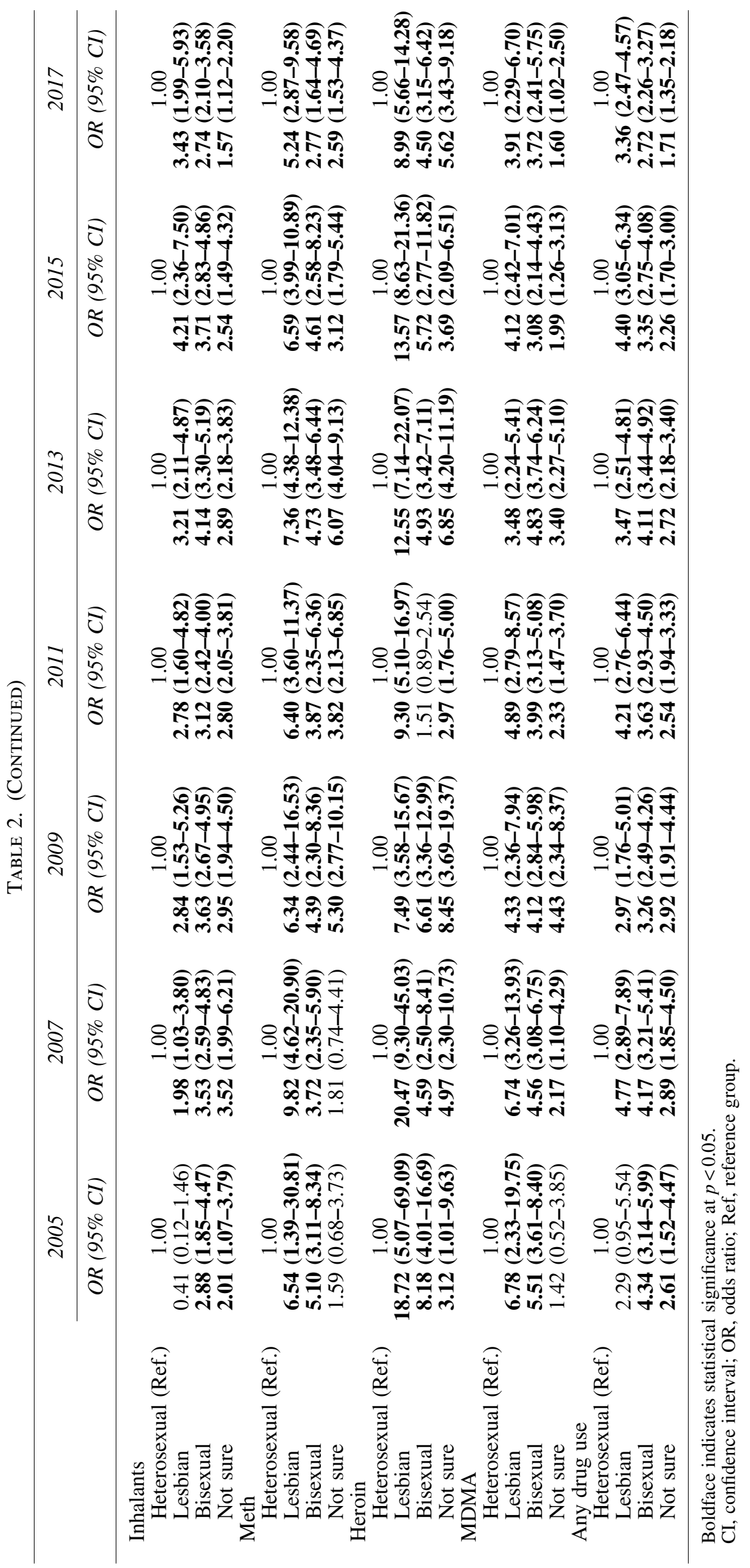


disparities within our results across seven different biennial survey administrations, the narrowing of disparities observed in that research could potentially represent a snapshot of fluctuation similar to what we observed. This then raises critical questions. Unless bisexual populations have begun to experience notably less stress than their gay or lesbian counterparts, an interpretation for which there is entirely no evidence, then minority stress alone may not account for these patterns. Indeed, prior research has established that bisexual populations may be at greater risk for minority stress than gay or lesbian populations, due to experiencing marginalization and stigma from both heterosexual and gay/lesbian groups. ${ }^{27}$ Therefore, these findings emphasize a need for targeted research to understand differential patterns of substance use among bisexual youth populations, taking into account the role of known protective and risk factors for youth substance use such as parental support, ${ }^{28}$ victimization, ${ }^{8}$ internalized stigma, ${ }^{29}$ community engagement, ${ }^{30}$ and social pressures. ${ }^{31}$

Our results follow a similar pattern as trend analyses of other drugs and alcohol. For example, as mentioned earlier, prior analyses of alcohol use behaviors have shown an overall decrease in alcohol use among all youth, with the greatest decreases observed among bisexual youth, although with the caveat that disparities persist despite this decline. ${ }^{23}$ A separate trend analysis of alcohol use among SMY demonstrated overarching decreases with the greatest declines seen among lesbian and bisexual female youth, with disparities again persistent across the decline. ${ }^{22}$ Analyses of changes in cigarette smoking behaviors from 2005 to 2015 demonstrated a narrowing of disparities for lesbian female youth and male bisexual youth but not for other sexual minority or unsure youth. ${ }^{32}$ Finally, an omnibus trend analysis of alcohol, tobacco, and marijuana use among Massachusetts youth found that disparities in these substances persisted from 1999 to 2013, and narrowed among male youth while persisting or widening among female youth. ${ }^{33}$ The similarities between these trends and those we observed affirm our results, while also highlighting the need for ongoing work to support in-depth research and targeted intervention to ensure equity in drug use outcomes for SMY.

Fortunately, prior study has illuminated potential intervention pathways. Victimization among youth-which is especially common among SMY, ${ }^{34}$ and which may contribute to experiences of minority stress, ${ }^{35}$ has been regularly linked to increased levels of alcohol and drug use in both sexual minority and sexual majority populations. ${ }^{8,36-38}$ Conversely, one of the most powerful protective factors available to sexual minority adolescents is a supportive school, social, and home environment. ${ }^{39-42}$ Therefore, interventions targeting school climate, familial support, and reduction of victimization are likely to be crucial to closing the gap between sexual minority and majority students' outcomes, and existing interventions of this nature should continue to be rigorously evaluated and-when effective-widely implemented. In the meantime, clinicians and school guidance counselors should consider regular screening and referral for substance use among SMY. Critically, given the known roles of stigma and minority stress in SMY substance use, school-based screening, referral, and/or resources should prioritize nonjudgmental, trauma-informed, and affirming practice.

Although there is evidence to support these preventive approaches, future research is still needed to better understand the multilevel drivers behind the trends in substance use that we and others have observed. Pairing quantitative analyses such as these with rigorous qualitative study may be helpful in more clearly illuminating causal pathways between minority stress and substance use, and how these may be changing over time.

Additional multilevel trend analyses which incorporate policy will also be critical. Policy changes such as the introduction of anti-LGB legislation may alter the degree of victimization, stigma, and minority stress experienced by marginalized individuals, therefore either increasing or decreasing illicit drug use, depending on the policy's psychosocial impact. ${ }^{17,43}$ Given the pervasive and structural causes of stigma and stress that lead to the elevated levels of drug use seen in SMY, ${ }^{15}$ assessing jurisdictional-level differences in use by various policy indicators of LGB acceptance/rejection will allow us to understand what policies are successful in reducing stigma, minority stress, and/or drug use on a wider scale, and should be implemented widely. Finally, future research should prioritize developing diverse samples to allow for analyses which are attentive to intersectional experiences of minority stress on the basis of oppression focused on race, ethnicity, gender, sex, and more. ${ }^{44,45}$

\section{Limitations}

There are limitations to our study. First, our analyses did not disaggregate data by state or city, although this was adjusted for by controlling for jurisdiction. This may mask jurisdictional differences in drug use risk. ${ }^{15}$ Furthermore, small sample sizes in certain drug use within certain years resulted in wide CIs, which present a challenge to confident interpretation. Future work should emphasize oversampling from marginalized minority groups to eliminate this concern. In addition, we were unable to directly account for minority stress in our analyses due to limitations in the available data. Future research which directly incorporates minority stress should be conducted to confirm our interpretations.

\section{Conclusions}

Although drug use generally declined from 2005 to 2017 , analyses of disparities within years revealed significant fluctuation, and statistically significant disparities remained between SMY and heterosexual youth in all years. Bisexual students demonstrated more significant downward trends than any other sexual minority group, a finding which is not readily explained and deserves additional attention. As interpreted in light of the minority stress framework, these results indicate that stigma and discrimination persist and continue to influence substance use disparities among SMY; however, factors other than minority stress are likely at play in creating the patterns we observed, and additional research is necessary to confirm this relationship.

\section{Disclaimer}

The content is solely the responsibility of the authors and does not necessarily represent the official views of the National Institutes of Health. In addition, this article is based on secondary data analysis. The authors wish to acknowledge the CDC for their role in creating and administering the YRBS. 


\section{Author Disclosure Statement}

No competing financial interests exist.

\section{Funding Information}

This study was funded by the National Institute on Alcohol Abuse and Alcoholism (R01 AA024409; principal investigator [PI]: G.P.), the National Heart, Lung, and Blood Institute (K12 HL143959; PI: L.B.B.), and the National Institute on Drug Abuse (K08 DA037825; PI: M.B., K01 DA039804A; PI: M.P.). One hundred percent of project costs were supported by these federal funding sources.

\section{Supplementary Material}

Supplementary Table S1

Supplementary Table S2

Supplementary Table S3

Supplementary Table S4

\section{References}

1. Johnston LD, Miech RA, O’Malley PM, et al.: Monitoring the Future National Survey Results on Drug Use 19752019: Overview, Key Findings on Adolescent Drug Use. Ann Arbor, MI: Institute for Social Research, 2020.

2. Feinstein EC, Richter L, Foster SE: Addressing the critical health problem of adolescent substance use through health care, research, and public policy. J Adolesc Health 2012; 50:431-436.

3. Corliss HL, Rosario M, Wypij D, et al.: Sexual orientation and drug use in a longitudinal cohort study of U.S. adolescents. Addict Behav 2010;35:517-521.

4. Li DH, Turner BC, Mustanski B, Phillips 2nd GL: Sexual orientation disparities in prescription drug misuse among a nationally representative sample of adolescents: Prevalence and correlates. Addict Behav 2018;77:143-151.

5. Marshal MP, Friedman MS, Stall R, et al.: Sexual orientation and adolescent substance use: A meta-analysis and methodological review. Addiction 2008;103:546-556.

6. McCabe SE, Hughes TL, Bostwick WB, et al.: Sexual orientation, substance use behaviors and substance dependence in the United States. Addiction 2009;104:1333-1345.

7. Newcomb ME, Birkett M, Corliss HL, Mustanski B: Sexual orientation, gender, and racial differences in illicit drug use in a sample of US high school students. Am J Public Health 2014;104:304-310.

8. Phillips 2nd G, Turner B, Salamanca P, et al.: Victimization as a mediator of alcohol use disparities between sexual minority subgroups and sexual majority youth using the 2015 National Youth Risk Behavior Survey. Drug Alcohol Depend 2017;178:355-362.

9. Hughes TL, Eliason M: Substance use and abuse in lesbian, gay, bisexual and transgender populations. J Prim Prev 2002;22:263-298.

10. Kann L, McManus T, Harris WA, et al.: Youth Risk Behavior Surveillance-United States, 2017. MMWR Surveill Summ 2018;67:1-114.

11. Medley G, Lipari RN, Bose J, et al.: Sexual Orientation and Estimates of Adult Substance Use and Mental Health: Results from the 2015 National Survey on Drug Use and Health. Rockville, MD: Substance Abuse and Mental Health Services Administration, 2016.
12. Kecojevic A, Jun HJ, Reisner SL, Corliss HL: Concurrent polysubstance use in a longitudinal study of US youth: Associations with sexual orientation. Addiction 2017;112: 614-624.

13. Meyer IH: Minority stress and mental health in gay men. J Health Soc Behav 1995;36:38-56.

14. Meyer IH: Prejudice, social stress, and mental health in lesbian, gay, and bisexual populations: Conceptual issues and research evidence. Psychol Bull 2003;129:674-697.

15. Hatzenbuehler ML, Jun HJ, Corliss HL, Bryn Austin S: Structural stigma and sexual orientation disparities in adolescent drug use. Addict Behav 2015;46:14-18.

16. Duncan DT, Hatzenbuehler ML, Johnson RM: Neighborhoodlevel LGBT hate crimes and current illicit drug use among sexual minority youth. Drug Alcohol Depend 2014;135: 65-70.

17. Hatzenbuehler ML, McLaughlin KA, Keyes KM, Hasin DS: The impact of institutional discrimination on psychiatric disorders in lesbian, gay, and bisexual populations: A prospective study. Am J Public Health 2010;100:452-459.

18. Fish JN, Watson RJ, Porta CM, et al.: Are alcohol-related disparities between sexual minority and heterosexual youth decreasing? Addiction 2017;112:1931-1941.

19. Fish JN, Watson RJ, Gahagan J, et al.: Smoking behaviours among heterosexual and sexual minority youth? Findings from 15 years of provincially representative data. Drug Alcohol Rev 2019;38:101-110.

20. Talley AE, Turner B, Foster AM, Phillips 2nd G: Sexual minority youth at risk of early and persistent alcohol, tobacco, and marijuana use. Arch Sex Behav 2019;48:1073-1086.

21. Johnston LD, Miech RA, O'Malley PM, et al.: Monitoring the Future National Survey Results on Drug Use, 19752018: Overview, Key Findings on Adolescent Drug Use. Ann Arbor, MI: Institute for Social Research, 2019.

22. Phillips II G, Turner B, Felt D, et al.: Trends in alcohol use behaviors by sexual identity and behavior among high school students, 2007-2017. J Adolesc Health 2019;65: 760-768.

23. Fish JN, Baams, L: Trends in alcohol-related disparities between heterosexual and sexual minority youth from 2007 to 2015: Findings from the Youth Risk Behavior Survey. LGBT Health 2018;5:359-367.

24. Centers for Disease Control and Prevention, Brener ND, Kann L, et al.: Methodology of the Youth Risk Behavior Surveillance System-2013. MMWR Recomm Rep 2013; 62:1-20.

25. Division of Adolescent and School Health, National Center for HIV/AIDS, Viral Hepatitis, STD, and TB Prevention, Centers for Disease Control and Prevention: 2015 YRBS Data User's Guide. Atlanta, GA: Centers for Disease Control and Prevention, 2016.

26. Centers for Disease Control and Prevention: Youth Risk Behavior Surveillance System (YRBSS): Conducting Trend Analyses of YRBS Data. Atlanta, GA, 2016. Available at https://www.cdc.gov/healthyyouth/data/yrbs/pdf/ 2015/2015_yrbs_conducting_trend_analyses.pdf Accessed September 18, 2020.

27. Friedman MR, Dodge B, Schick V et al.: From bias to bisexual health disparities: Attitudes toward bisexual men and women in the United States. LGBT Health 2014;1:309-318.

28. Ryan C, Huebner D, Diaz RM, Sanchez J: Family rejection as a predictor of negative health outcomes in White and Latino lesbian, gay, and bisexual young adults. Pediatrics 2009; 123:346-352. 
29. Willoughby BLB, Doty ND, Malik NM: Victimization, family rejection, and outcomes of gay, lesbian, and bisexual young people: The role of negative GLB identity. J GLBT Fam Stud 2010;6:403-424.

30. Phillips II G, Felt D, McCuskey D, et al.: Engagement with LGBTQ community moderates the association between victimization and substance use among a cohort of sexual and gender minority individuals assigned female at birth. Addict Behav 2020;107:106414.

31. Jordan KM: Substance abuse among gay, lesbian, bisexual, transgender, and questioning adolescents. School Psychol Rev 2000;29:201-206.

32. Fish JN, Turner B, Phillips 2nd G, Russell ST: Cigarette smoking disparities between sexual minority and heterosexual youth. Pediatrics 2019;143:e20181671.

33. Watson JR, Porta C, Adjei J, Saewyc E: Substance use among sexual minorities: Has it actually gotten better? Subst Use Misuse 2018;53:1221-1228.

34. Poteat VP, Birkett M, Turner B, et al. Changes in victimization risk and disparities for heterosexual and sexual minority youth: Trends from 2009 to 2017. J Adolesc Health 2020; 66:202-209.

35. Mustanski B, Andrews R, Puckett JA: The effects of cumulative victimization on mental health among lesbian, gay, bisexual, and transgender adolescents and young adults. Am J Public Health 2016;106:527-533.

36. Birkett M, Espelage DL, Koenig B: LGB and questioning students in schools: The moderating effects of homophobic bullying and school climate on negative outcomes. J Youth Adolesc 2009;38:989-1000.

37. Hughes T, McCabe SE, Wilsnack SC: Victimization and substance use disorders in a national sample of heterosexual and sexual minority women and men. Addiction 2010;105: 2130-2140.

38. Hughes TL, Johnson TP, Steffen AD, et al.: Lifetime victimization, hazardous drinking, and depression among heterosexual and sexual minority women. LGBT Health 2014;1: 192-203.
39. Moody RL, Starks TJ, Grov C, Parsons JT: Internalized homophobia and drug use in a national cohort of gay and bisexual men: Examining depression, sexual anxiety, and gay community attachment as mediating factors. Arch Sex Behav 2018;47:1133-1144.

40. Ryan C, Russell ST, Huebner D, et al.: Family acceptance in adolescence and the health of LGBT young adults. J Child Adolesc Psychiatr Nurs 2010;23:205-213.

41. Davis B, Royne Stafford MB, Pullig C: How gay-straight alliance groups mitigate the relationship between gay-bias victimization and adolescent suicide attempts. J Am Acad Child Adolesc Psychiatry 2014;53:1271.e1-1278.e1.

42. Heck NC, Livingston NA, Flentje A, et al.: Reducing risk for illicit drug use and prescription drug misuse: High school gay-straight alliances and lesbian, gay, bisexual, and transgender youth. Addict Behav 2014;39:824-828.

43. Raifman J, Moscoe E, Austin SB, McConnell M: Differencein-differences analysis of the association between state same-sex marriage policies and adolescent suicide attempts. JAMA Pediatr 2017;171:350-356.

44. Bauer GR: Incorporating intersectionality theory into population health research methodology: Challenges and the potential to advance health equity. Soc Sci Med 2014;110: 10-17.

45. Bowleg L: The problem with the phrase women and minorities: Intersectionality-an important theoretical framework for public health. Am J Public Health 2012;102:1267-1273.

Address correspondence to: Dylan Felt

Department of Medical Social Sciences Northwestern University Feinberg School of Medicine 625 N. Michigan Avenue, Suite 1400 Chicago, IL 60611 USA E-mail: dylan.felt@northwestern.edu 